\title{
Fingerprint Enhancement by Shape Adaptation of Scale-Space Operators with Automatic Scale
}

\section{Selection}

Andrés Almansa and Tony Lindeberg

EDICS Categories 2-NFLT, 2-WAVP, 2-LFLT

A. Almansa is with the Scientific Computing Centre, School of Engineering, University of the Republic of Uruguay, 11300 Montevideo, Uruguay (e-mail: almansa@fing.edu.uy).

T. Lindeberg is with the Department of Numerical Analysis and Computing Science, KTH (Royal Institute of Technology), S-100 44 Stockholm, Sweden (e-mail: tony@nada.kth.se).

This work has been supported by BID-CONICYT project number 96/94, by the BID-CONICYT's human resources program by the SIDA/BITS collaboration program between KTH and the Engineering School at the University of Uruguay, and by the Swedish Research Council for Engineering Sciences, TFR. An earlier version of this work was presented in [1], and an extended technical report [2] is available from http://www.nada.kth.se/cvap/abstracts/cvap216.html. 


\begin{abstract}
This work presents two mechanisms for processing fingerprint images; shape-adapted smoothing based on second moment descriptors and automatic scale selection based on normalized derivatives.

The shape adaptation procedure adapts the smoothing operation to the local ridge structures, which allows interrupted ridges to be joined without destroying essential singularities such as branching points and enforces continuity of their directional fields. The scale selection procedure estimates local ridge width and adapts the amount of smoothing to the local amount of noise. In addition, a ridgeness measure is defined, which reflects how well the local image structure agrees with a qualitative ridge model, and is used for spreading the results of shape adaptation into noisy areas.

The combined approach makes it possible to resolve fine scale structures in clear areas while reducing the risk of enhancing noise in blurred or fragmented areas. The result is a reliable and adaptively detailed estimate of the ridge orientation field and ridge width, as well as a smoothed grey-level version of the input image.

We propose that these general techniques should be of interest to developers of automatic fingerprint identification systems as well as in other applications of processing related types of imagery.
\end{abstract}

\title{
Keywords
}

Fingerprint, enhancement, scale-space, affine, diffusion, automatic scale selection, image processing.

\section{INTRODUCTION}

Fingerprint enhancement is a common step in several systems for automatic fingerprint identification $[3],[4],[5],[6],[7]$. Such systems usually mimic the human procedure for fingerprint identification, consisting of the following processing steps:

- The image is analyzed in local neighborhoods, to estimate attributes of the ridge patterns, such as ridge width, orientation and curvature, as well as the amount of noise and the image quality.

- Then, a set of contextual filters is built, based on this local information, with the aim of enhancing the underlying ridge structure while removing the noise.

- Once a clean image has been obtained, the minutiae features (i.e. singularities in the ridge structure such as ridge endings and bifurcations) can be extracted from the image, as well as their attributes (usually type and orientation) and relationships (such as ridge count between two minutiae). These features are eventually post-processed, in order to reduce the number of false positives.

- Finally the detected features are coded into a feature vector, which is either stored in a database or compared (matched) to other vectors in the database to find a correspondent if it exists.

Traditional works on fingerprint enhancement [3], [8], [9], [10], [11] performed local estimates as well as contextual filtering in a highly discretized manner. These methods often resulted in blocky artifacts in 
the output as well as in poor estimates of the local image characteristics. Such artifacts may introduce several problems in a later feature detection phase, and hence many mechanisms have been proposed to avoid them [12], [13], [14]. Among these approaches, the most flexible one consists of estimating orientations by means of a structure tensor or second moment descriptor [15], [16], [17], [7] and by substituting the contextual filtering step by a special kind of anisotropic diffusion scheme [18], [15], [16], which was first proposed for fingerprint image processing in [16]. These ideas have to be further developed in order to design a fully automatic and highly accurate module for image enhancement.

In this work, we develop new mechanisms to perform shape adapted smoothing (section II) in a way that better reflects the orientations of the ridges. We also present a set of scale-selection mechanisms (section III) that can be used to produce a continuous and reliable estimate of the local distance between ridges. In addition, a ridgeness measure is defined in section IV, which reflects how well the local image structure agrees with a qualitative ridge model, and is shown to play a central role for the shape adaptation and scale selection mechanisms. Section V shows a possible way to combine the proposed mechanisms into a composed algorithm for image enhancement. Finally, sections VI and VII give experimental results, which illustrate the qualitative properties of the proposed methods as well as a quantitative evaluation of their performance. The presentation ends with a comparison with similar approaches (section VIII), as well as a summarized assessment of the results obtained (section IX).

\section{SHAPE-ADAPTED SMOOTHING}

\section{A. Linear scale-space}

The method we shall propose for enhancing a fingerprint image $f: \mathbb{R}^{2} \rightarrow \mathbb{R}$ is based on shape adaptation of scale-space operators in linear scale-space. This scale-space representation is defined as a one-parameter family of 2-D signals $L: \mathbb{R}^{2} \times \mathbb{R}_{+} \rightarrow \mathbb{R}$ that satisfies the diffusion equation

$$
\partial_{t} L=\frac{1}{2} \nabla^{\mathrm{T}} \nabla L=\frac{1}{2} \operatorname{div}(\nabla L)
$$

with initial condition $L(\mathbf{x} ; 0)=f(\mathbf{x})$. This linear scale-space representation satisfies several uniqueness properties [19], [20], [15], [21], [22], and is a natural framework to use as pre-processing for feature detectors expressed in terms of differential geometric image measurements [15], [21].

\section{B. Affine Gaussian scale-space}

When dealing with strongly non-isotropic images (as in the case of fingerprint images), one can relax the rotational invariance assumption underlying the formulation of isotropic linear scale-space, while still preserving most of the essential scale-space properties [18], [15], [23], [21], [16]. A similar situation 
occurs when dealing with affine transformations of the image domain, as shape from texture and shape from disparity gradients [24], [23]. Relaxing the rotational invariance assumption in this way leads to the affine Gaussian scale-space representation generated by the anisotropic diffusion equation ${ }^{1}$

$$
\partial_{t} L\left(\cdot ; \Sigma_{t}\right)=\frac{1}{2} \nabla^{\mathrm{T}} \Sigma_{0} \nabla L\left(\cdot ; \Sigma_{t}\right)
$$

with initial condition $L(\cdot ; 0)=f$, where $\Sigma_{0}$ is a symmetric positive semi-definite matrix, and $\Sigma_{t}=t \Sigma_{0}$ is a multiple of the initial diffusion matrix. For notational convenience, we also denote this solution by

$$
\mathcal{D}_{\Sigma_{t}} f=L\left(\cdot ; \Sigma_{t}\right)
$$

or by $\mathcal{D}_{t} f=L(\cdot ; t)$, when the diffusion matrix is isotropic and hence $\Sigma_{t}=t I$.

\section{The second moment matrix and shape adaptation}

When the diffusion matrix $\Sigma_{0}$ is the identity, equation (2) reduces to the linear scale-space representation (1). When dealing with anisotropic images, however, we shall adapt the diffusion matrix $\Sigma_{0}$ to the local image structure as measured by a second moment matrix [25], [26], [27], [28], [29], [30], [31]

$$
\mu\left(\cdot ; \Sigma_{t}, s\right)=\mathcal{D}_{s}\left(\nabla L\left(\cdot ; \Sigma_{t}\right) \nabla L\left(\cdot ; \Sigma_{t}\right)^{\mathrm{T}}\right)
$$

computed as the exterior product of the gradient vectors by themselves in the affine Gaussian scalespace representation $L\left(\cdot ; \Sigma_{t}\right)$ of $f$ at local scale $t$, subsequently diffused up to the integration scale $s$. By expanding the tensor product

$$
\mu=\left(\begin{array}{ll}
\mu_{11} & \mu_{12} \\
\mu_{12} & \mu_{22}
\end{array}\right)=\mathcal{D}_{s}\left(\begin{array}{cc}
L_{x}^{2} & L_{x} L_{y} \\
L_{x} L_{y} & L_{y}^{2}
\end{array}\right)
$$

and introducing the descriptors

$$
P=\mu_{11}+\mu_{22}=\mathcal{D}_{s}\left(L_{x}^{2}+L_{y}^{2}\right), \quad C=\mu_{11}-\mu_{22}=\mathcal{D}_{s}\left(L_{x}^{2}-L_{y}^{2}\right), \quad S=2 \mu_{12}=2 \mathcal{D}_{s}\left(L_{x} L_{y}\right)
$$

it can be seen that $P \geq 0$ indicates the local image contrast, $Q=\sqrt{C^{2}+S^{2}} \in[0, P]$ measures the degree of anisotropy and $\theta=\frac{\pi}{2}+\frac{1}{2} \tan ^{-1} \frac{S}{C} \in[0, \pi]$ reflects the dominant orientation (the orientation most orthogonal to the gradients in a neighborhood of $\mathbf{x}$, which will be used for estimating ridge orientation).

If we interpret the symmetric positive definite matrix $\mu$ as a quadratic form, the corresponding ellipse $\left\{\xi \in \mathbb{R}^{2}:(\xi-\mathbf{x})^{\mathrm{T}} \mu(\mathbf{x})^{-1}(\xi-\mathbf{x})=1\right\}$, will be elongated in the direction orthogonal to $\theta$, with eccentricity $(1+\tilde{Q}) /(1-\tilde{Q})$, where $\tilde{Q}=Q / P \in[0,1]$ is the normalized anisotropy. Specifically, multiples of $\mu^{-1}$

${ }^{1}$ Throughout this treatment we consider continuous data. The transfer to discrete images of finite support is straightforward, if we follow the guidelines in [15, chapters 4 and 5]. 
constitute natural choices for scale matrices, since we want a larger amount of smoothing along ridges than across them. Computation of $\mu$, however, requires determination of a local scale matrix $\Sigma_{t}$. In [15], [24], [23] it was proposed that it is natural to look for a fixed point $\Sigma$ such that

$$
\mu\left(\cdot ; \Sigma_{t}, s\right)=\Sigma^{-1}, \quad \Sigma_{t}=t \Sigma
$$

and it was shown that a similar fixed point is preserved under affine transformations. The process of reaching such a fixed point is referred to as shape adaptation. Simple iteration of the fixed point condition yields a linearly convergent algorithm. In practice, when applied to fingerprint images, the convergence rate is close to 2 , and starting from a rotationally symmetric $\Sigma_{0}=I$ about 5 iterations suffice to obtain a $1 \%$ accurate approximation to the fixed point $\Sigma[2]$.

Strictly, the preservation property of fixed points under affine transformations requires anisotropic integration smoothing, given by an integration scale matrix $\Sigma_{s}=s \Sigma$ proportional to $\Sigma$ at the fixed point. When implementing this scheme in practice, the second stage integration smoothing corresponds to the largest computational work, while the local scale matrix causes the largest effect in practice. Hence, to improve the computational efficiency, we use isotropic integration scale matrices, i.e., $\Sigma_{s}=s I$.

\section{Enhancement of ridges by shape adaptation}

When expressing a shape adaptation procedure in practice, it turns out that a number of technical problems have to be solved.

1. One question concerns how to express a scalar size descriptor of a possibly non-isotropic diffusion matrix. Experiments on several fingerprint images [2] showed that the minor axis of the ellipse is a better choice than, for example, the major axis or the square root of the ellipse area, and we define

$$
\Sigma_{t}=t \frac{\Sigma}{\|\Sigma\|_{\lambda_{\min }}}
$$

where $t$ is the scalar size descriptor and $\|\Sigma\|_{\lambda_{\min }}$ represents the minor axis of the ellipse defined by $\Sigma$. This choice ensures a minimum amount of smoothing as a means of noise suppression [15], and provides a consistent interpretation of the scale parameter across several degrees of anisotropy [2, appendix C]. The latter property is essential when combining shape adaptation with a scale selection procedure.

2. Another technical problem arises in highly anisotropic regions, where $\Sigma$ becomes near singular, and shape adaptation may lead to an exaggerated amount of blurring in some direction. To avoid this effect, we use a regularized diffusion matrix (having the same eigenvectors)

$$
\Sigma=(\mu+\varepsilon I)^{-1}
$$


From the eigenvalues of $\mu$, we have

$$
\operatorname{cond}(\Sigma)=\operatorname{cond}(\mu+\varepsilon I)=1+\frac{2 Q}{(2 \varepsilon+P-Q)} .
$$

Since $(P-Q) \geq 0$, it follows that $\operatorname{cond}(\Sigma) \leq 1+\frac{Q}{\varepsilon}=\kappa$ and the natural choice

$$
\varepsilon=\frac{Q}{(\kappa-1)}
$$

ensures a uniform (user supplied) upper bound $\kappa$ on the eccentricity of $\Sigma$, preventing ellipses from becoming too elongated. Imposing such a bound is also necessary if we want the discretized scale-space representation to preserve scale-space properties of its continuous counterpart [32], and significantly improves the performance of certain numerical methods for computing it [2, appendix B].

3. At any stage it is essential that the integration scale is larger than the local scale, i.e.:

$$
s \geq \beta^{2}\left\|\Sigma_{t}\right\|
$$

for some $\beta>1$. Failure to satisfy this relation might lead to unreliable estimates of the ridge directions when computing $\mu$ for shape adaptation, which in turn might lead to undesirable enhancement of spurious directional fine-scale structures. Whereas in [1] $\|\cdot\|=\operatorname{det}$ was used in (12) to allow for finescale shape adaptation near singularities (as minutiae), a more detailed study revealed that $\|\cdot\|=\lambda_{\min }$ allows for more reliable shape adaptation results in noisy areas, when complemented by a more careful (automatic) tuning of the safety factor $\beta$ (to be described in section IV).

\section{Automatic SCAle Selection}

When applying the shape adaptation procedure in practice, another important problem concerns how to choose suitable values for the local scale parameter $t$ and the integration scale parameter $s$ when computing the second moment descriptor (4). A good choice of these parameter values is always related to the distance between ridges in the fingerprint.

Ideally, the local scale should be selected in such a way that the gradient vectors at that scale reflect the local ridge orientation. This parameter should be sufficiently large to suppress the influence of noise and other fine-scale structures, while it should be small enough to avoid the shape distortion effects that occur if too much smoothing is used. In practice, the choice of this parameter is determined by the relationship between the size of the object and the local noise level.

The integration scale defines the size of the neighborhood over which statistics of gradient directions is computed. Ideally, this parameter should be as small as meaningfully possible if we expect to discriminate rapid variations in contrast, anisotropy or orientation. In particular, small integration 
$s=t$
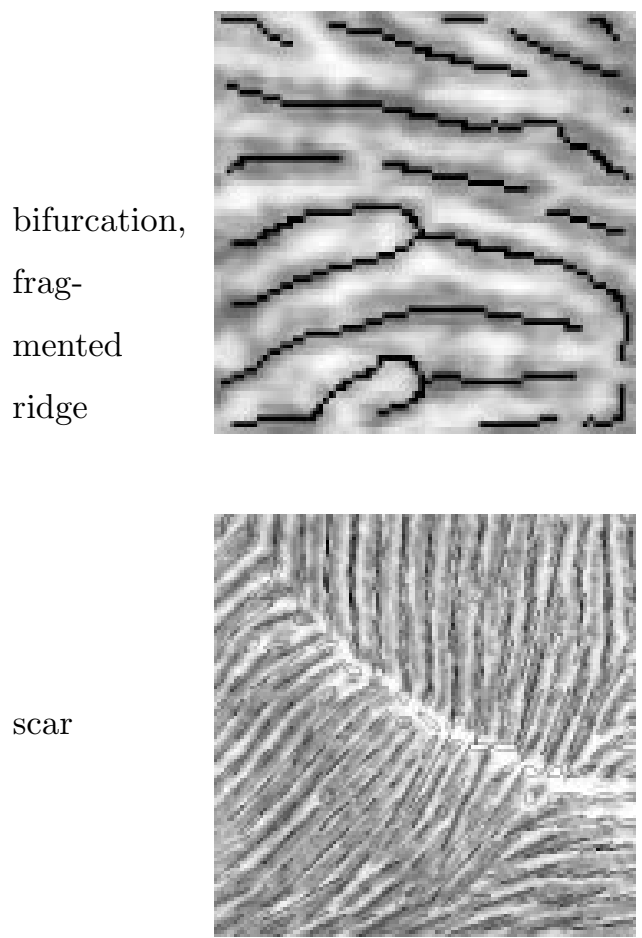

$s=2^{2} t$
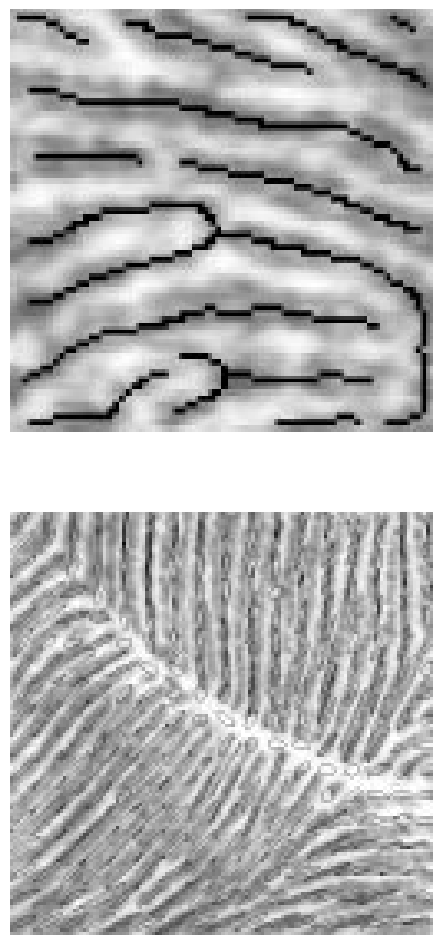

$s=8^{2} t$
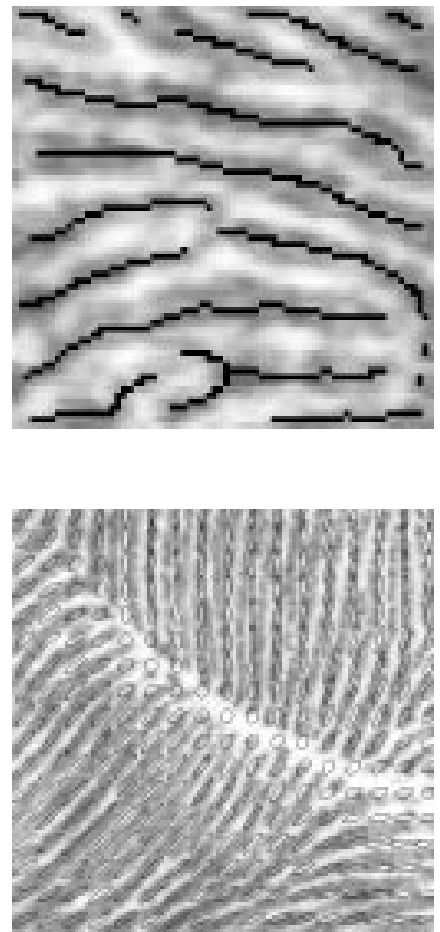

Fig. 1. The need for automatic selection of integration scales. This figure shows an example where different values of the integration scale parameter $s$ are required in different parts of the image. The bifurcation in the center of the top-row images can only be captured with a small to medium value of $s$, whereas the continuity of the fragmented ridge can only be enforced with a large value of $s$. Similarly, in the presence of a scar, as shown in the bottom-row images, larger values of $s$ are needed to avoid a $\pi / 2$ error in the orientation field.

scales are needed to resolve the rapid variations that occur in ridge directions around minutiae. The presence of noise, however, calls for larger neighborhoods to reduce the error of our estimators. In particular, large integration scales are needed to bridge gaps in regions of poor fingerprint structures. It is also important that the consistency requirement (12) is satisfied.

Notably, suitable values of these scale parameters may take very different values in different fingerprints (e.g., due to age, resolution or finger differences), and may vary substantially even within the same fingerprint (see figure 1 for an illustration of the practical influence of these parameters). Hence, to tune these scale levels, explicit mechanisms for automatic scale selection are required.

\section{A. Ridge width estimates from differential descriptors}

A powerful approach to perform local and adaptive selection of scales for feature detection is by detecting local extrema over scales of normalized differential descriptors [33], [15], [34], [35]. Our design of a specific scheme for this application will be based on the following general results: 
- If certain natural requirements are imposed on a scale selection methodology based on local maxima over scales, such as preservation of local maxima over scales under size variations (a form of scale invariance), then it can be proved that the corresponding differential descriptors should be expressed in terms of $\gamma$-normalized derivatives [34] where $\gamma$ is a free parameter (to be determined later)

$$
\partial_{\xi}=t^{\gamma / 2} \partial_{x}
$$

- If we consider the following local model of the ridge structure

$$
f(u, v)=A \sin \frac{2 \pi}{\lambda} v
$$

which corresponds to a cylindrical sine wave of amplitude $A$ and wavelength $\lambda$, then any $m$-th order $\gamma$-normalized derivative assumes a single local maximum over scales at scale $t_{\max }$ with [34]

$$
\lambda=2 \pi \sqrt{\frac{t_{\max }}{\gamma m}} .
$$

This expression provides a direct link between scales $t_{\max }$, at which local maxima over scales are assumed, and estimated ridge widths $\lambda$. In the following, these ideas will be further developed and we will design a set differential descriptors, which are specifically tuned to the selection of local scales and integration scales for processing fingerprint images.

\section{B. Local scale selection from $Q_{\text {norm }}$}

In related work on shape from texture and disparity gradients [15], [30], local maximization of $\tilde{Q}$ over scales was used for automatic selection of local scales. Here, we shall multiply this anisotropy measure by the contrast measure $P$, expressed in terms of normalized derivatives with $\gamma=1$, and thus maximize the normalized anisotropy measure

$$
Q_{\text {norm }}=t P \tilde{Q}=t \sqrt{C^{2}+S^{2}}
$$

over scales. The underlying idea is to select scale levels where the following criteria hold simultaneously:

- the local image structure should be highly non-isotropic, corresponding to a high value of $\tilde{Q}$, and

- the scale value should reflect the size of the dominant image structures, corresponding to a high value of $\mathcal{P}_{\text {norm }}=t P$.

Given a local maximum $t_{Q_{\text {norm }}}$ of $Q_{\text {norm }}$ over scales, the wavelength is thus estimated according to

$$
\lambda_{Q_{\mathrm{norm}}}=2 \pi \sqrt{t_{Q_{\mathrm{norm}}}} .
$$


Extensive experiments show that this approach produces good wavelength estimates on a large set of fingerprint images. This scale selection scheme, however, also introduces an additional scale problem, namely that of choosing a suitable integration scale $s$ for computing $Q_{\text {norm }}$. Since a specific choice of integration scale $s$ implies that only a certain range of local scales $t$ are meaningful, we can only make a sensible choice in this respect if we already have a reasonable estimate of the relevant range of scales.

To provide an initial estimate of integration scale for such a bootstrapping step, we will in the next section present a method that computes such an estimate using local descriptors only, i.e., differential geometric descriptors depending on pointwise derivatives only. Such descriptors also have the additional advantage that they require less computational work than $Q_{\text {norm}}$.

\section{Scale selection from quasi quadrature pairs}

In [34], [36], [1] it was proposed that local frequency estimation can be performed by pointwise maximization of the following quasi quadrature measure

$$
\mathcal{P}_{\text {norm }} L=t\left(L_{x}^{2}+L_{y}^{2}\right)+C t^{2}\left(L_{x x}^{2}+L_{y y}^{2}+2 L_{x y}^{2}\right)
$$

over scales. This differential expression is a rotationally symmetric differential invariant, which measures the energy in the first- and second-order derivatives. With $C \approx 2 / 3$, it approximates the local power spectrum for a signal with one-dimensional symmetry. Moreover, this expression can be seen as an approximation of a quadrature pair, within the Gaussian derivative framework.

For the purpose of processing fingerprint images, however, it turns out that this expression, in addition to elongated fingerprint structures, also responds to spatially isotropic noise. To express a scale selection mechanism that is more specifically sensitive to ridge-like structures, we shall therefore replace the second-order isotropic energy measure $L_{x x}^{2}+L_{y y}^{2}+2 L_{x y}^{2}$ by the directionally more sensitive ridge-strength measure $\mathcal{A}_{\gamma-\text { norm }}=t^{2 \gamma}\left(\left(L_{x x}-L_{y y}\right)^{2}+4 L_{x y}^{2}\right)$ [37]. Moreover, we shall choose the normalization of the derivatives in such a way that the normalization of the first-order edge strength measure $\mathcal{G}_{\gamma-\text { norm }} L=$ $t^{\gamma}\left(L_{x}^{2}+L_{y}^{2}\right)$ and second-order ridge strength measure $\mathcal{A}_{\gamma-\text { norm }}$ agree with the normalization values for edge detection and ridge detection proposed in [37], [35]. This leads to the combined measure

$$
\mathcal{S}_{\text {norm }} L=\mathcal{G}_{\gamma_{1}-\text { norm }} L+C \mathcal{A}_{\gamma_{2}-\text { norm }}
$$

with $C=2 / 3, \gamma_{1}=\frac{1}{2}$ and $\gamma_{2}=\frac{3}{4}$. In other words,

$$
\mathcal{S}_{\text {norm }} L=t^{\frac{1}{2}}\left(L_{x}^{2}+L_{y}^{2}\right)+C t^{\frac{3}{2}}\left(\left(L_{x x}-L_{y y}\right)^{2}+4 L_{x y}^{2}\right) .
$$

While the spatial response of this operator is rather insensitive to the local phase information (thereby the name "quasi quadrature measure"), the scales at which they are assumed have a strong phase 
dependency, depending on whether the first- or the second-order derivative information is dominant. (Equation (15) is not directly applicable, since the differential expression $\mathcal{S}_{\text {norm }}$ mixes first- and secondorder derivatives.) Therefore, we compute a first order approximation of the wavelength by considering the relative contribution of first and second order parts to $\mathcal{S}_{\text {norm }}$, at the scale $t_{\mathcal{S}_{\text {norm }}}$ at which this measure is maximized. In other words, we let

$$
\lambda_{\mathcal{S}_{\text {norm }} L}=2 \pi \sqrt{\frac{t_{\mathcal{S}_{\text {norm }}}^{\gamma m}}{\gamma m}} \quad \text { where } \quad \gamma m=\left.\frac{\gamma_{1} m_{1} \mathcal{G}_{\gamma_{1}-\text { norm }} L+\gamma_{2} m_{2} C \mathcal{A}_{\gamma_{2}-\text { norm }} L}{\mathcal{S}_{\text {norm }} L}\right|_{t=t_{\mathcal{S}_{\text {norm }}}} .
$$

\section{Suppression of multiple maxima}

Experiments show that the abovementioned approach gives distinct peaks in the scale-space signature, corresponding to the width of the ridge-like structures in the fingerprints. The scale selection mechanism can, however, also give rise to multiple local maxima over scales, typically due to noise responses at fine scales. In the general case, an explicit mechanism is required for extracting the local maxima corresponding to the desired structures. Here, the following simplified procedure is applied:

- An average scale is computed by $\bar{t}_{\mathcal{M}}=\operatorname{argmax}_{t} \int_{\mathbb{R}^{2}} \mathcal{M}$. If the scale-space signature of $\mathcal{M}$ at a certain point $x$ has multiple local maxima, then the maximum closest to $\bar{t}_{\mathcal{M}}$ is selected.

- The selected scales are constrained to the interval $\left[\bar{t}_{\mathcal{M}} / T, \bar{t}_{\mathcal{M}} T\right]$, where $T \approx 4$.

- The map of the selected scales is smoothed spatially with a median filter with its size $n \bar{\lambda}$ equal to an integer multiple of the average wavelength.

- Scale selection is performed in the shape-adapted affine Gaussian scale-space.

The choice of $T \approx 4$ is motivated by the fact that the variations in fingerprint ridge width are smooth, and usually within a factor of 2 for a single image. Spurious responses of $\mathcal{S}_{\text {norm }}$ usually occur as isolated impulse noise, and can be suppressed by a median filter, if the size of this filter is chosen in concordance with the periodic phase dependency effects. Finally, by performing scale selection in the anisotropic scale-space, spurious responses caused by interference between nearby ridges can be removed.

Figure 2 shows the result of applying these two methods for automatic scale selection to a synthetic fingerprint image with continuously varying ridge widths, as well as a collage of two fingerprint structures. Observe, for both measures, the ability of the scale selection method to capture the variations in ridge widths, even under extreme noise conditions and sudden changes in ridge width and contrast.

\section{E. Selection of local scales and integration scales}

Once a local scale $t$ has been determined according to section III-B, the next step is to determine the integration scale. Referring to the consistency requirement $s \geq \beta^{2} t$ and the experimental results in 

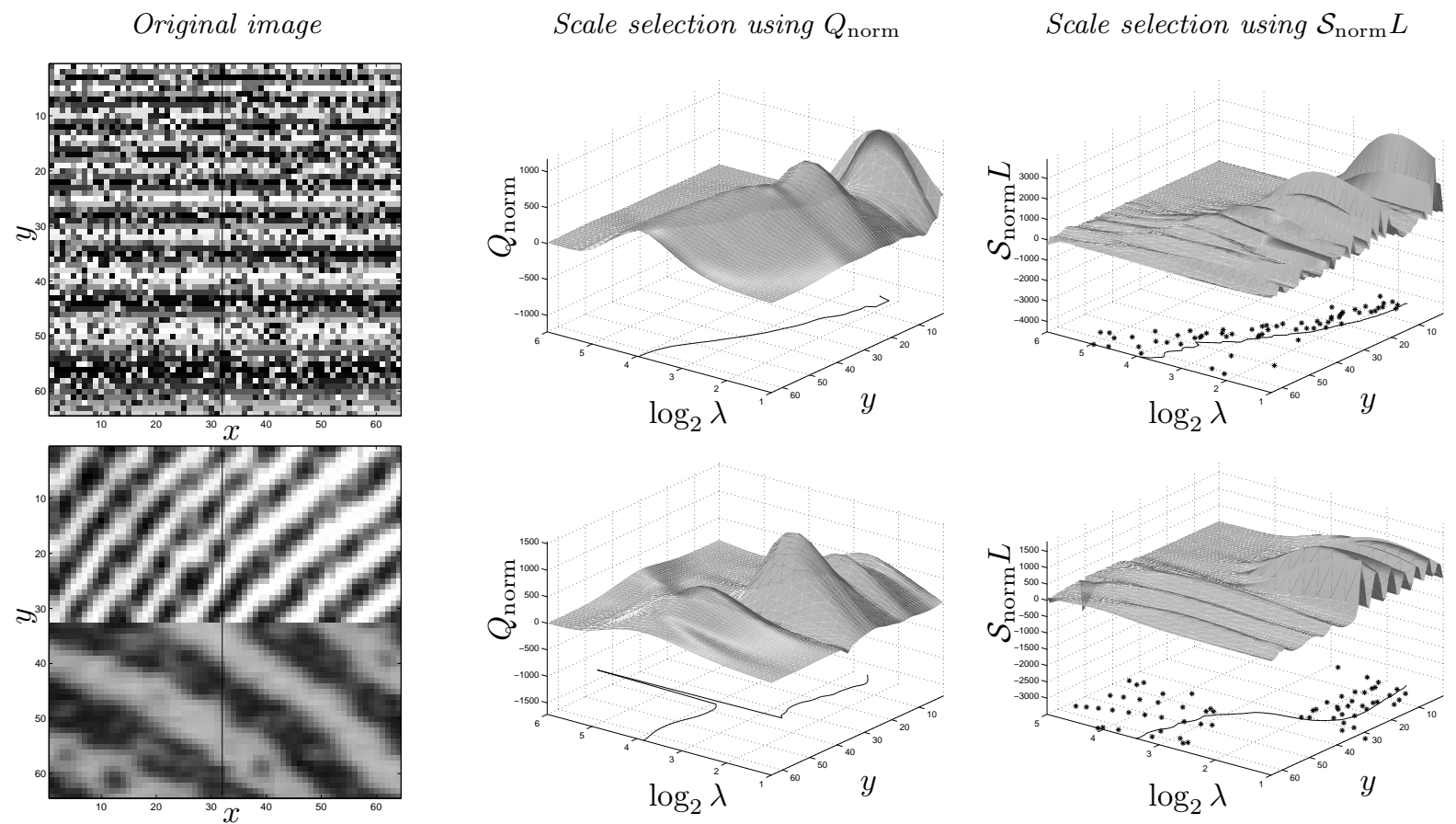

Fig. 2. Automatic scale selection by maximizing $Q_{\text {norm }}$ and $\mathcal{S}_{\text {norm }}$ over scales. The left column shows an image with wavelengths varying from 4 to 16 pixels with $60 \%$ speckle noise and a collage of two fingerprint structures having ridge widths of 7 and 14 pixels respectively. In the middle column, the signatures of $Q_{\text {norm }}$ along a vertical cross-section at $x=32$ are shown as functions of scale for different values of the vertical $y$-coordinate, thus forming a surface. The curve below the surface represents the scale at which the signatures assume local maxima over scales. Similarly, the right column shows the signatures of $\mathcal{S}_{\text {norm }} L$ as a surface. In this case, the scales at which the local maxima over scales are assumed are represented as stars, whereas the continuous curve represents the scales obtained after median filtering of the scale map. In both cases, the scale axis has been normalized according to equation (15) to express the distance $\lambda$ between ridges in logarithmic scale. Observe how both measures reflect the variations in ridge width, even under extreme noise conditions and sudden changes in ridge width and contrast.

Figure 1 for different values of $\beta$, we can see that $\beta=\beta_{\text {good }}=1$ is a reasonable value only in very clear areas, whereas $\beta=\beta_{\text {poor }}=8$ is required to obtain reliable orientation estimates in extremely noisy areas. In transitional areas, $\beta=\beta_{\text {trans }}=2$ is a reasonable compromise.

To continuously tune $\beta$ to the local image quality, we introduce a ridgeness measure $\mathcal{Q}: \mathbb{R}^{2} \rightarrow[0,1]$, such that $\mathcal{Q}=\mathcal{Q}_{\text {poor }}=0$ in poor areas and $\mathcal{Q}=\mathcal{Q}_{\text {good }}=1$ in good ones. Assuming that $\mathcal{Q}=\mathcal{Q}_{\text {trans }}$ is an optimal threshold between these two kinds of regions, $\beta$ is then chosen as function of $\mathcal{Q}$, by interpolating these three points according to

$$
\beta=\beta_{\text {good }} b^{(1-\mathcal{Q})^{a}} \quad \text { where } \quad b=\frac{\beta_{\text {good }}}{\beta_{\text {poor }}} \text { and } \quad a=\log _{\left(1-\mathcal{Q}_{\text {trans }}\right)} \log _{b} \frac{\beta_{\text {trans }}}{\beta_{\text {good }}} .
$$


The next section defines such a discriminatory function $\mathcal{Q}$, which is also useful for several other purposes.

\section{Ridgeness meAsure}

The previous section motivates the need of a ridgeness measure $\mathcal{Q}: \mathbb{R}^{2} \rightarrow[0,1]$, defined for every point of the input image such that it:

- takes values close to 1 in areas with clear parallel ridges, even if these areas contain minutiae,

- takes values close to 0 whenever the local image structure does not correspond to ridges, such as e.g. background, fragmented ridges, scars, blurred, damaged, over- and under-inked areas,

- is slightly dependent on contrast, giving a lower response for low-contrast ridges that may be confused with background texture or quantization effects.

One way to define such a ridgeness measure is by:

$$
\mathcal{Q}=\min \left(1, \sqrt{\frac{P}{P_{\max }}} \tilde{Q}\right)
$$

The normalized anisotropy $\tilde{Q}$ is always in the range $[0,1]$ and characterizes anisotropic structures like ridges. This measure should be computed at the local scale $t=\overline{t_{Q_{\text {norm }}}}$, and at an integration scale $s=8^{2} \overline{t_{Q_{\text {norm }}}}$ to make it sufficiently localized, while at the same time being insensible to small-scale isotropic ridge structures, like minutiae or pores.

The factor $\sqrt{P / P_{\max }}$ gives the ridgeness measure a slight dependency on contrast, and this factor is rarely larger that 1 , if we regard

$$
P_{\max }=\frac{A^{2}}{4 t e}
$$

as a "pragmatic" upper bound for $P$, derived under the assumption that the input signal is a sine wave (14) with amplitude $A$ and wavelength $\lambda=2 \pi \sqrt{t}$, where $t$ is the local scale level for computing $P$ (i.e. $\left.t=\overline{t_{Q_{\text {norm }}}}\right)$. For certain extreme signals, like a pure square wave, $P$ may assume slightly higher values than $P_{\max }$, but for real fingerprints this hardly happens. Rather, $P$ is very close to $P_{\max }$ in clean areas. The amplitude $A$ of the underlying ridge model is, in turn, estimated from the local image variance as:

$$
\hat{A}=\sqrt{2\left(\mathcal{D}_{s}\left(f^{2}\right)-\left(\mathcal{D}_{s} f\right)^{2}\right)}, \quad A=\hat{A}+A_{\min } \frac{A_{\max }-\hat{A}}{A_{\max }}
$$

where $A_{\max }=256$ and $A_{\min }=16$ depend on the dynamic range of the input image, and the importance of background noise and quantization effects.

A qualitative and quantitative evaluation (see section VI-B) shows that the proposed ridgeness measure reasonably well satisfies our design requirements, and that it can be used for selecting integration scales as described in section III-E. 
The ridgeness measure is also useful for other purposes. For example, the shape adaptation procedure can be improved by substituting equation (4) by a weighted second moment descriptor

$$
\mu\left(\cdot ; \Sigma_{t}, s, \mathcal{Q}\right)=\frac{\mathcal{D}_{s}\left(\nabla L\left(\cdot ; \Sigma_{t}\right) \nabla L\left(\cdot ; \Sigma_{t}\right)^{\mathrm{T}} \mathcal{Q}\right)}{\mathcal{D}_{s} \mathcal{Q}}
$$

where lower weight is given to gradient estimates in low quality regions. This modification effectively reduces the typical $\pi / 2$ orientation error near scars (illustrated in figure 1 ). More generally, it increases the reliability of the orientation estimates and the eccentricity of the diffusion matrix, which leads to improved ridge connectivity in fragmented areas, as illustrated in figure 3. Other applications of this measure are discussed in section VI-B.

\section{Composed Algorithm}

The proposed mechanisms for shape adaptation and scale-selection can be used in various ways within enhancement and identification systems. For the purpose of experiments and validation, we have here integrated these modules into a composed algorithm, according to the following four-step procedure:

Stage 1 constructs, in the absence of further information, the isotropic scale-space (1) for a large range of scales. Then, it finds an average local scale $\bar{t}_{\text {local }}^{(1)}$ and wavelength $\bar{\lambda}^{(1)}$, by maximizing $\int_{\mathbb{R}^{2}} \mathcal{S}_{\text {norm }} L$ over scales, and using (21).

These scale values are used for constraining further analysis to the scale range $\left[\bar{\lambda}^{(1)} / 2,2 \bar{\lambda}^{(1)}\right]$, and for computing the ridgeness measure $\mathcal{Q}$ at local scale $\bar{t}_{\text {local }}^{(1)}$ and integration scale $s=8^{2} \bar{t}_{\text {local }}^{(1)}$.

$\underline{\text { Stage } 2}$ constructs a first orientation estimate $\mu^{(2)}$, by iterating the $\mathcal{Q}$-weighted shape adaptation procedure (7) and (26) with spatially constant local scales and integration scales as in stage 1 . Then $\mathcal{S}_{\text {norm }} L$ is maximized over scales in the resulting affine Gaussian scale-space, which after application of (21) results in a pointwise local scale map $t_{\text {local }}^{(2)}$.

$\underline{\text { Stage } 3}$ refines the results of the previous stage by performing shape adaptation with spatially varying local scales $t_{\text {local }}^{(2)}$, and scale selection based on $Q_{\text {norm }}$. This produces a refined orientation estimate $\mu^{(3)}$ and our final local scale map $t_{\text {local }}^{(3)}$.

$\underline{\text { Stage } 4}$ refines the orientation estimate by using local scales $t=t_{\text {local }}^{(3)}$, and spatially varying integration scales $s=\beta^{2} t$. This results in our final orientation estimate $\mu^{(4)}$, and enhanced image $L\left(\cdot ; \Sigma_{t_{\text {local }}^{(3)}}^{(4)}, \beta^{2} t_{\text {local }}^{(3)}\right)$.

In the above procedure, the $\mathcal{Q}$-weighted shape adaptation procedure (used in stages 2, 3 and 4 ) consists of the following steps (assuming known local scale $t$, integration scale $s$ and initial estimate of $\mu$ ):

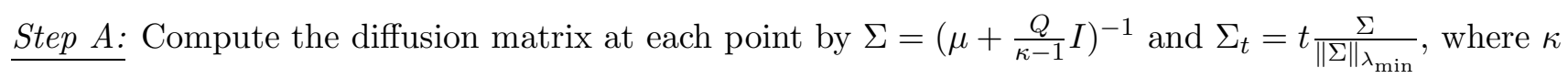
is an upper bound on the eccentricity of $\Sigma$. 
Proposed algorithm
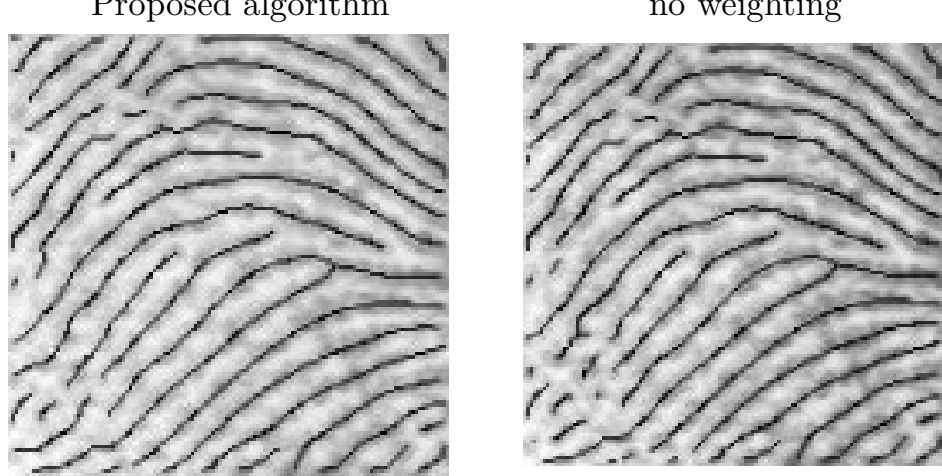

no weighting

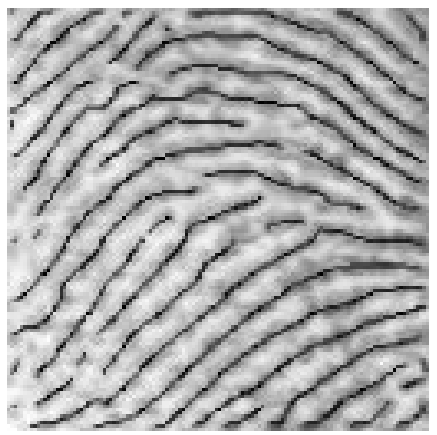

scale

coarse in-

tegration

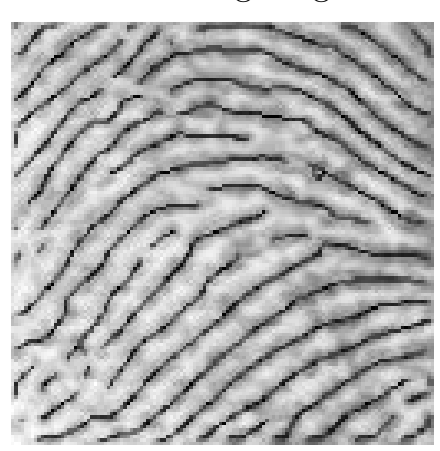

fixed $t=\bar{t}$

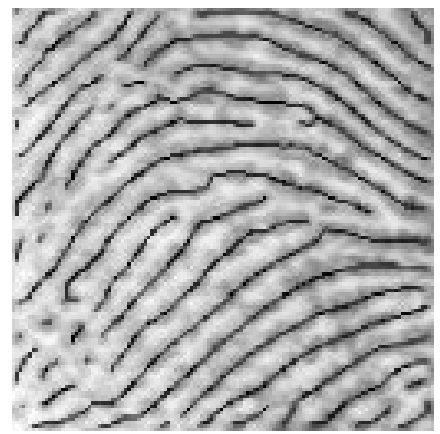

fixed $t=\bar{t}$

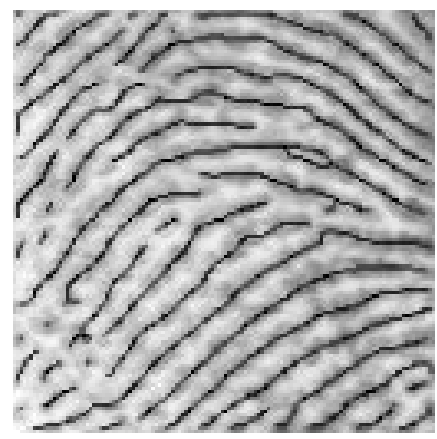

no weighting

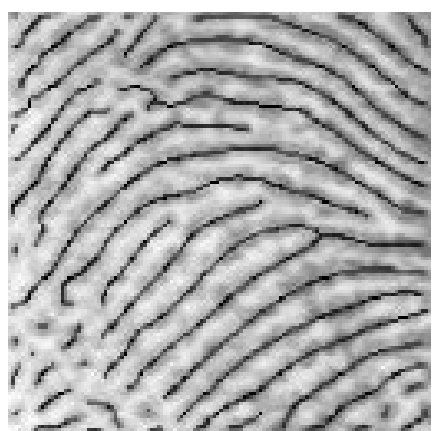

fixed $t=\bar{t}$

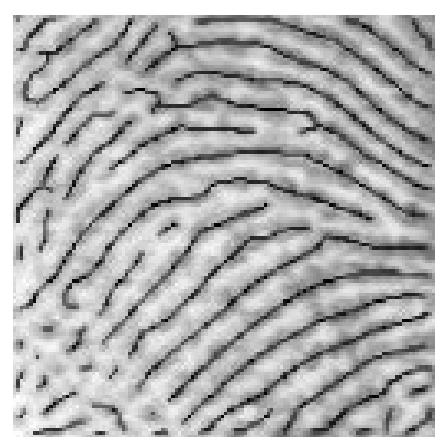

Fig. 3. Illustration of the effects of different modules in the composed shape adaptation and scale selection algorithm. The output of the proposed method (top left) is compared to the result of running the last stage without some of the proposed features. The top row shows the result of applying the last stage with the same local and integration scales as in the proposed method, but without ridgeness-based weighting during shape-adaptation (central column); or with a fixed local scale (right column). The central and bottom rows illustrate the result of using fixed integration scales set to the maximum and minimum values allowed in the proposed algorithm, instead of the proposed scale selection scheme. Observe how the quality of the computed skeleton is rapidly degraded when any of the proposed features is omitted. In particular, observe the ability of the proposed method to spread the correct estimates of the orientation field into the fragmented lower left area, which results in more continuous ridges. 


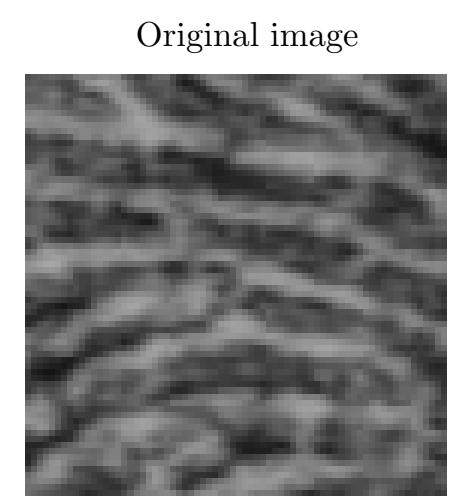

Stage 1

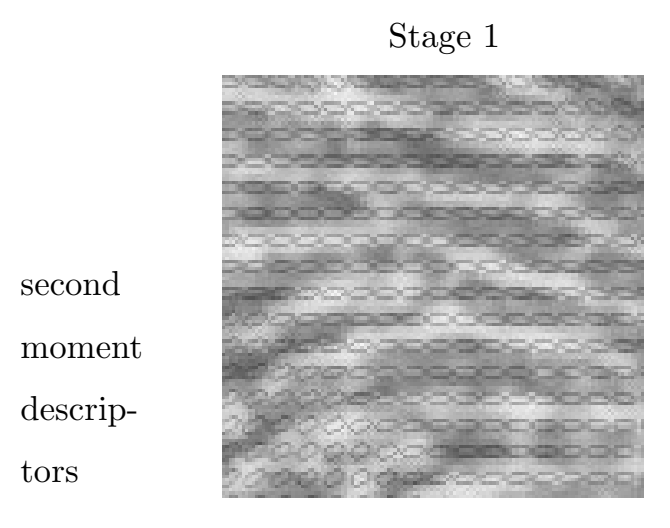

tors

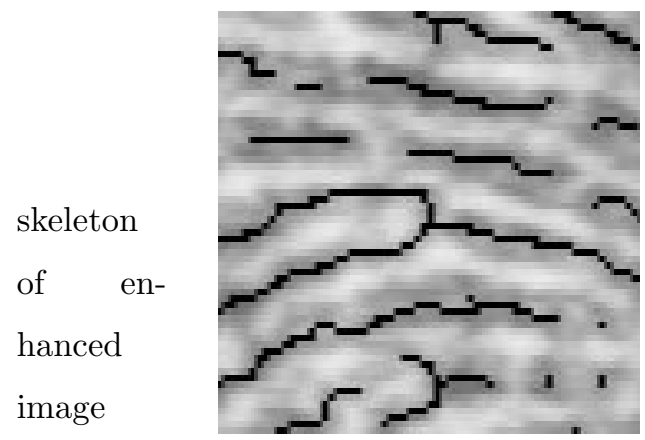

Selected scales at stage 2

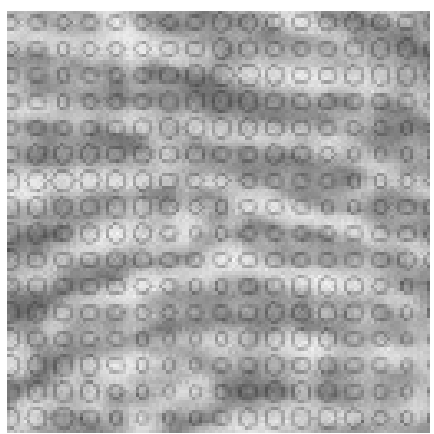

Stage 2
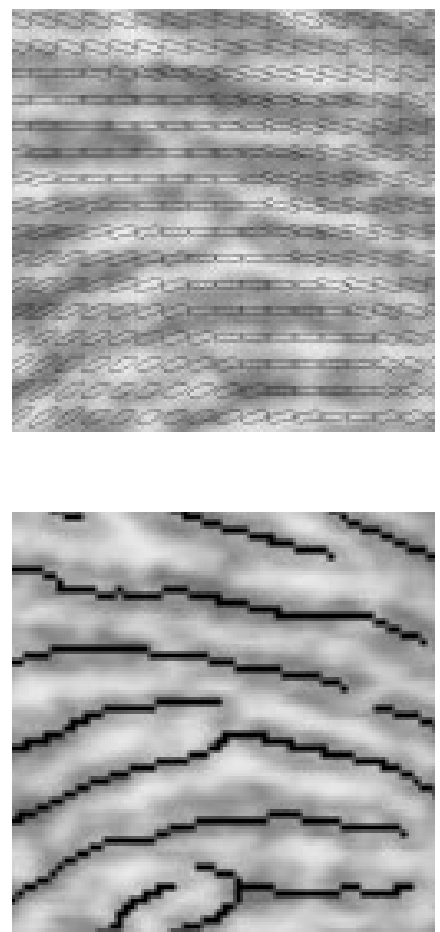

Stage 4
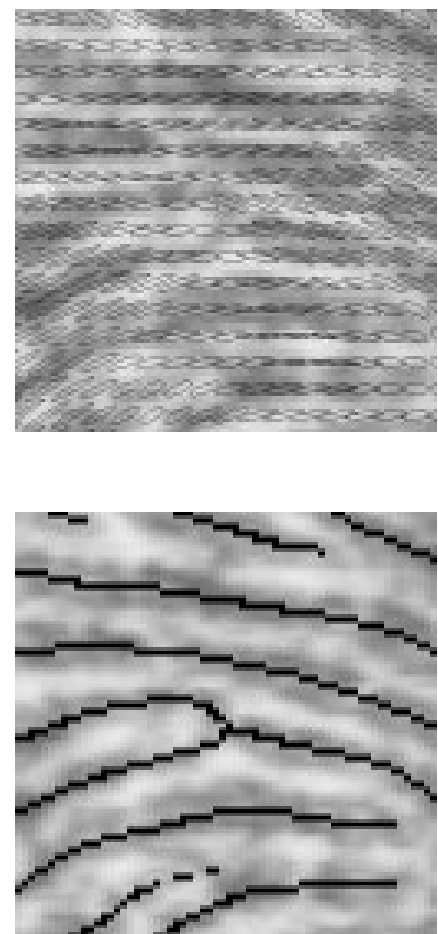

Fig. 4. Illustration of the various stages in the composed shape adaptation and scale-selection algorithm. Stage 1 uses isotropic smoothing and allows the preservation of the central bifurcation at the cost of several interrupted lines as well as spurious small ridges. In stage 2, a globally constant coarse integration scale produces a coarse estimate of the orientation field, resulting in highly anisotropic second moment descriptors. This gives more continuous ridges and fewer spurious responses at the cost of broken bifurcations. The coarse orientation field, which breaks the central bifurcation, does, however, not prevent the scale selection mechanism from capturing the rapid variations in ridge width around this point (see top row). After stage 3, which mainly refines the results of stage 2, the final stage 4 constructs an adaptively detailed orientation field, based on scale selection from stages 2 and 3. Observe how the total effect of these successive processing stages allows details to be preserved, while ridge lines are joined and spurious ridges eliminated. 
Step B: Construct the scale-space filtered image $L=\mathcal{D}_{\Sigma_{t}} f$ at scale $t$, and compute the weighted second moment descriptor $\mu^{\prime}=\frac{\mathcal{D}_{s}\left(\nabla L \nabla L^{\mathrm{T}} \mathcal{Q}\right)}{\mathcal{D}_{s} \mathcal{Q}}$.

Step C: If the relative change $\int_{\mathbb{R}^{2}} \frac{\left\|\mu^{\prime}-\mu\right\|}{\|\mu\|}$ is small enough, then $\mu^{\prime}$ (and the corresponding diffusion matrix $\Sigma^{\prime}$ is considered to have converged to the fixed point (7). Otherwise, return to step A with $\mu=\mu^{\prime}$ and loop until convergence is achieved in this step.

Here, $\mu=I$ is used as initial condition in the first stage, while the result of the previous stage are used in later stages. Furthermore, we initially set $\mathcal{Q}=1$, and let $s$ and $t$ be globally constant estimates. Later stages produce progressively more refined and spatially localized estimates of $s, t$, and $\mu$.

Figure 4 shows how this algorithm progressively reconstructs the fingerprint structures, and figure 3 illustrates the importance of each of the proposed mechanisms in the final result that is obtained.

It is worth emphasizing that this prototype algorithm does not only produce an enhanced image, but also pointwise estimates of ridge width, orientation and local image quality, which can be used in later stages of a fingerprint identification system.

\section{Experiments And Evaluation: I}

To evaluate the performance of the proposed mechanisms, we have applied the prototype algorithm in section $\mathrm{V}$ to a selected set of standard image databases and compared the output with the result of manual measurements by fingerprint recognition experts.

\section{A. Material}

For the evaluation, we used a subset of 10 fingerprints from NIST database 4 [38], 10 fingerprint pairs from NIST database 14 [39], and 14 fingerprint images used for evaluation in [40] (referred to as image set MM). These images were manually extended with the following information by a forensic expert:

- The position of all reliable minutiae, and their type (ridge ending, bifurcation or transition).

- A set of points where the image quality is good (clear parallel ridges, where the minutiae can be clearly distinguished), and a set of points where the image quality is poor (scars, fragmented ridges, blurred or over-inked areas, which make it more difficult to distinguish real minutiae from noise).

In addition, we provided the following information by counting the number of ridges (typically between 4 and 8) within a circle of fixed diameter

- The average ridge width within a neighborhood of each point on a regular grid. This grid was later interpolated to obtain the ridge width at each point.

Given the tedious work that this represents, not all information was obtained for all images. Minutiae were obtained for the data sets MM (yielding 567 minutiae in 14 images) and NIST14 (yielding 1969 
minutiae in 20 images), whereas image quality was estimated for set NIST4 (yielding 379 points of both classes in 10 images), and ridge width only for the image in the NIST4 set, judged to have the largest internal ridge width variations.

\section{B. Evaluation of the ridge width estimates}

For the image in NIST database 4, having internal ridge width variations of $100 \%$, the manually measured distance between ridges was compared to the automatically selected scales, by means of the relative differences $\left(\lambda_{\mathcal{S}_{\text {norm }}} / \lambda-1\right)$ and $\left(\lambda_{Q_{\text {norm }}} / \lambda-1\right)$. In both cases, we obtained a negative bias of $-10 \%$ and a standard deviation of $10 \%$, which is within the errors of the manual measurements (figure 5).

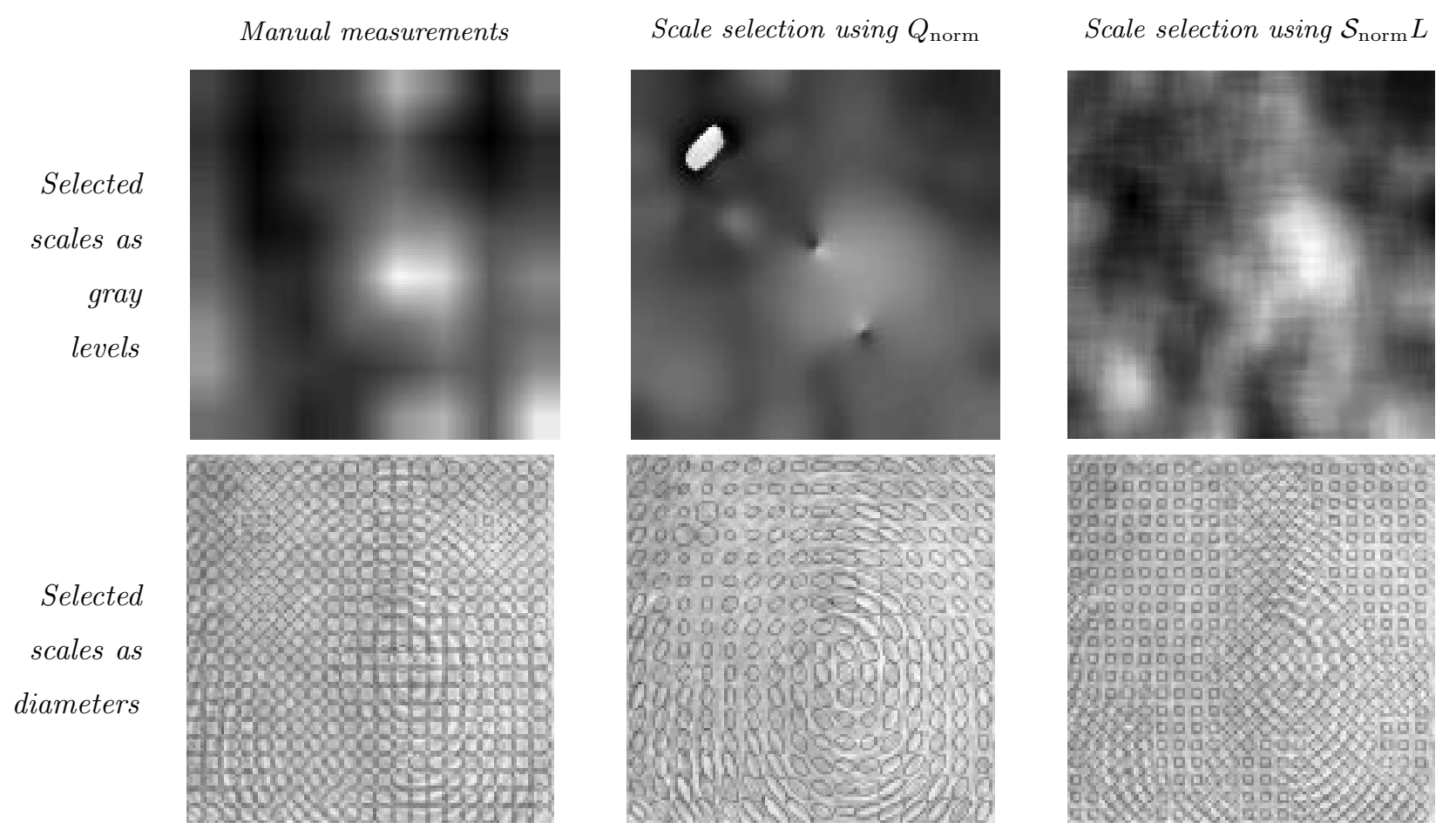

Fig. 5. Results of automatic scale selection by maximizing $Q_{\text {norm }}$ and $\mathcal{S}_{\text {norm }}$ over scales. In the upper row, brighter gray-levels represent coarser scales. In the lower row, the selected scales are represented by diameters of circles. In the case of $Q_{\text {norm }}$, the selected scale is reflected by the minor axis of the ellipse, while the orientation and the reflect the attributes of the second moment descriptor used for computing $Q_{\text {norm }}$ at the selected scale. Observe how the selected scales reproduce the corresponding manually determined pattern.

\section{Evaluation of the ridgeness measure}

A first qualitative study on several images (see figure 7 for a few samples) showed that the proposed ridgeness measure $\mathcal{Q}$ effectively discriminates good areas (taking values usually above 0.6), from scars, 
fragmented ridges, blurred and over-inked areas (taking values usually below 0.4 ). This property was unaffected by high or low global contrast, and even by smooth variations in local image contrast. The only undesired results were related to very small noisy areas (that are regarded as good areas) and highly curved areas, where the ridgeness measure always takes very small values.

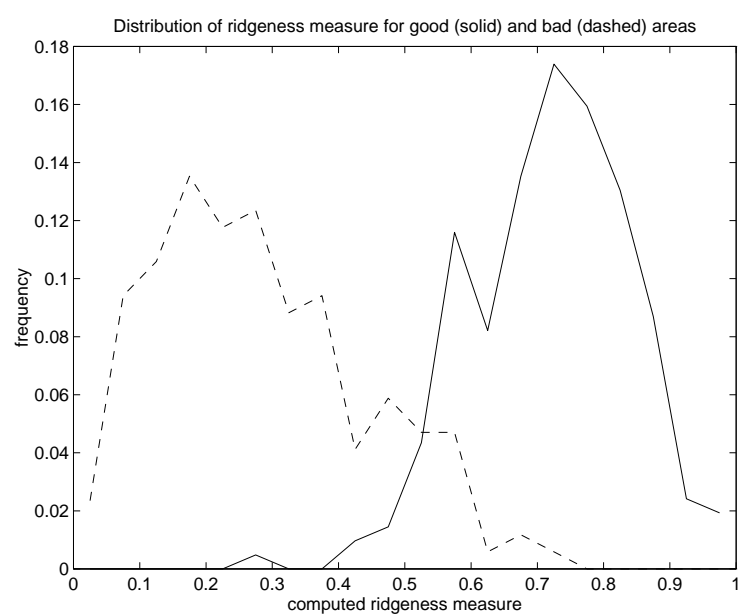

Fig. 6. Empirical distribution of the ridgeness measure. The solid curve represents the empirical distribution of ridgeness within good areas (parallel ridges and true minutiae), whereas the dashed curve represents the distribution within poor areas (scars, blurred, fragmented, over/under-inked). Observe the reasonable separation of the two classes.

A quantitative study was then conducted by comparing the ridgeness measure with the image quality information available for the NIST4 image set. This was done by comparing the histogram of $\mathcal{Q}$ over all areas classified as "good" by the forensic expert (shown as a solid line in figure 6), with the same histogram taken over all areas classified as "poor" (shown as a dotted line in figure 6). The results show that $93 \%$ of the class "poor" has a value of $\mathcal{Q}$ below 0.55 , and that $93 \%$ of the class "good" has a value of $\mathcal{Q}$ above this threshold. This suggests that $\mathcal{Q}$ reasonably well discriminates these two classes, which was the main design goal in section IV.

\section{Orientation and isotropy estimates}

Unfortunately, it is hard to carry out an objective evaluation of the quality of the orientation estimates. Besides the large amount of manual work required to produce dense orientation fields, it is not clear which orientation should be selected at branching points, and in general the manual choice of orientation will be dependent on the size of the neighbourhood presented to the operator. For these reasons, we restrict ourselves to describing qualitative properties of the computed orientation estimates.

- Orientation fields contain a coarse description in poor regions, where statistics are taken over larger neighborhoods, leading to low anisotropy values, which still allow orientation discrimination. 
- A much more detailed description is obtained in clear areas, where the rapid changes in orientation near branching points are captured, leading to highly anisotropic second moment descriptors at parallel ridges, and isotropic second moment descriptors (no preferred orientation) at the centers of minutiae.

- Scars are usually assigned orientations ranging between "no orientation" and the orientation of their surrounding ridges.

These properties can be observed in figure 4. Highly anisotropic second moment descriptors allow the connectivity of parallel ridges to be enforced, and may even break bifurcations. These properties can also be observed in the computed skeletons (see figure 3 ).

\section{EXPERIMENTS AND Evaluation: II}

To measure the accuracy of the enhanced gray-scale image is harder, in particular in the lack of a true reference. Here, we shall evaluate the performance of the composed prototype algorithm in an indirect way, by measuring to what extent the minutiae in the fingerprint structure can be captured, using a simple algorithm for minutiae extraction consisting of the following steps [41], [11], [42]:

- apply a local contrast adaptive threshold to the input gray-level image,

- apply a thinning algorithm to the resulting binary image,

- detect ridge endings and bifurcations by means of a 3x3 logical mask on the skeleton so obtained,

- postprocess the minutiae set by filtering out minutiae having a ridgeness value below a threshold.

It should be noted that this evaluation procedure has obvious limitations, since it measures the performance of the enhancement module with respect to a specific feature detection algorithm. One could conceive using more sophisticated and accurate feature detection algorithms, which are more closely adapted to and integrated with the pre-processing step. For example, since our initial developments [1] methods have been developed which take advantage of ridge width and orientation outputs in the feature detection step [43], perform differential ridge detection with automatic scale selection [35], and detect minutiae directly from grey-level data [40]. Another possibility could be by estimating the performance with respect to a matching algorithm [44]. The current evaluation procedure is chosen because of historical reasons, and its availability in the AFIS project this work is a part of.

\section{A. Definitions}

Given a set $D$ of minutiae obtained by the abovementioned minutiae extraction algorithm, our evaluation measures (following [40]) are based on comparing $D$ with the set $M$ of true minutiae marked 
by the forensic expert. For this purpose, we consider the following sets: ${ }^{2}$

- The set $F P=D-M$ of false positives or false minutiae.

- The set $F N=M-D$ of false negatives or missed minutiae.

- The set $D \cap M=C C \cup F C$ of correctly detected minutiae is partitioned into two sets: $C C$ of correctly classified minutiae, and FC of falsely classified minutiae, depending on whether the detected type (ridge ending or bifurcation) coincides or not with the type assigned by the expert.

\section{B. Results of minutiae detection}

Figure 7 shows a few results of applying the minutiae detection algorithm to the output of the composed prototype fingerprint enhancement algorithm. The results have been compared with the manual classification and been colour coded in the following way:

- "o" represents a ridge ending or any of its equivalent minutiae types (dot or island ending),

- "x" represents a bifurcation or any of its equivalents (fork, enclosure ending or bridge ending),

- "*" is a minutia for which it could not be decided whether it is a ridge ending or a bifurcation,

- green represents a correct minutia (in $C C$ ), i.e. one that was marked by the expert, found by the algorithm and correctly classified,

- yellow represents a misclassified minutia (in $F C$ ), i.e. correctly detected by the algorithm, while the type differs from the type selected by the expert,

- red represents a false positive (in FP), i.e. a minutia that has been detected by the proposed method but was not marked by the expert,

- blue represents a false negative, (in $F N$ ), i.e. a minutia that has been marked by the expert but not detected by the proposed method.

Results are shown for two images from the MM image set and one image from the NIST4 database. (As a general rule, the images in the MM image set have much higher image quality than the images in the NIST4 data base.) As can be seen from the illustrations, the output from the algorithm agrees rather well with the features marked by the expert in regions where the ridge structures are clear and approximately parallel. When features are missed, that typically occurs at extremely fine scale details such as dots and pores (see the center of image 5 from the MM set), which cannot be expected to be

${ }^{2}$ For points not coinciding exactly, we identify a point $d \in D$ with a point $m \in M$ if $d$ and $m$ are not too far apart, and no point $d^{\prime} \in D$ is closer to $m$ than $d$ and similarly for every point $m^{\prime} \in M$. In addition, we require the mapping between points in $D$ and $M$ to be one to one. For points marked as "transition" by the expert, meaning that the image does not provide enough information to make a decision on the minutiae type, the detected type is always regarded as correct. 
Post-processed minutiae over original

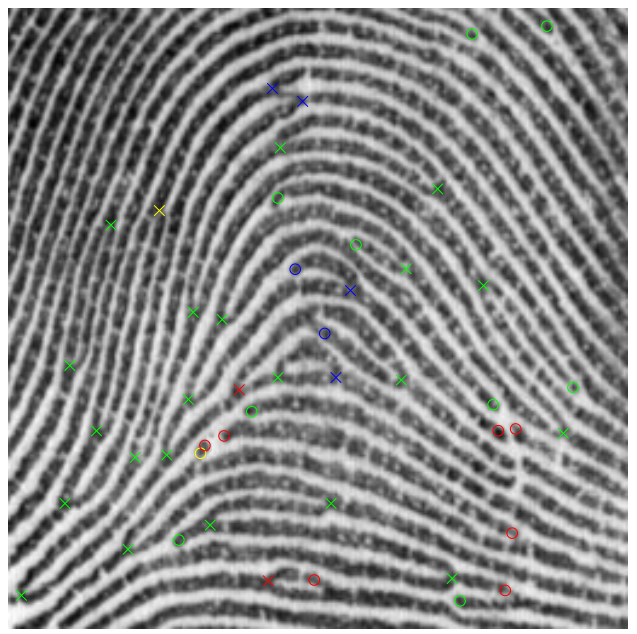

MM image 1

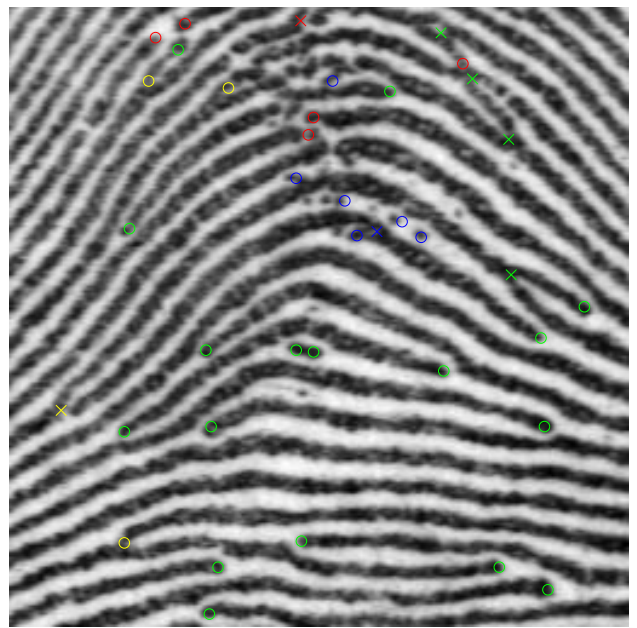

MM image 5

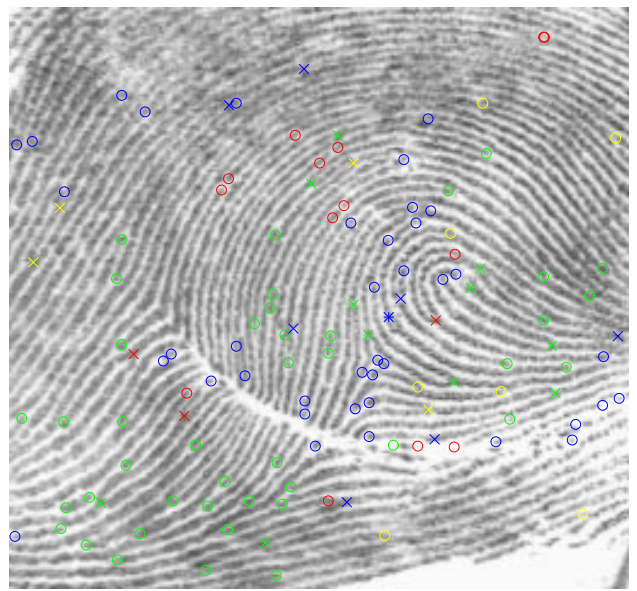

NIST 14 image f0000971
Minutiae before post-processing

over skeleton and thresholded ridgeness

(threshold values: $0.4,0.45,0.5,0.55,0.6$ )
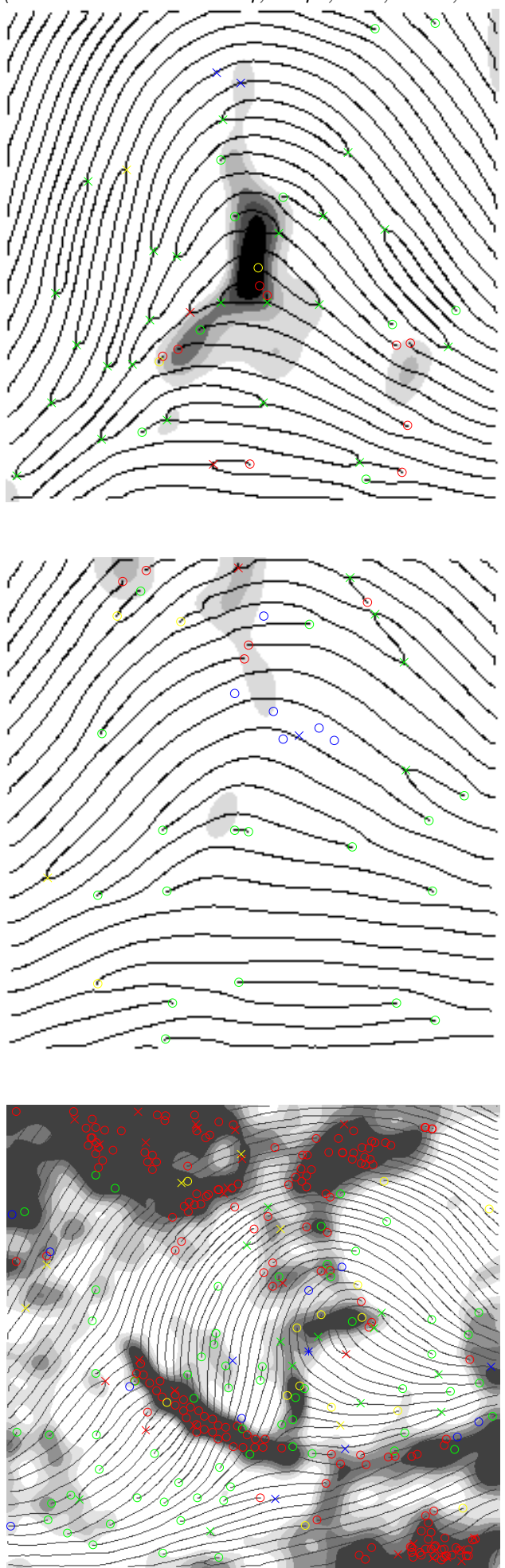

Fig. 7. Results of applying a ridge structure and minutiae detection algorithm to the output of the composed fingerprint enhancement algorithm. The figure shows the extracted minutiae overlayed on the original greylevel image, as well as an image showing the extracted ridge structure and thresholded ridgeness measure. (Symbols: "o" = ridge ending, "x" = bifurcation, "** = transition, green = fully correct, yellow = correctly detected but misclassified, red $=$ false positive, blue $=$ false negative. $)$. 
reliably detected by an automated system lacking explicit mechanisms for such features, or in regions where the ridge structures are highly curved (see the center of image 1 from the MM set). Besides the latter effect, post-processing by thresholding on the ridgeness measure effectively suppresses false positives due to background, scars and other noisy areas (see image f0000971 from the NIST 14 set). It is worth noting that many false positives are due to small scars that the ridgeness measure did not capture (see center-right of image 1 from the MM set), but more importantly due to apparent omissions of the expert (see the lower part of image 1 from the MM set). To make a more fair judgement about the algorithm, it would thus be preferable to use classifications from more than one fingerprint expert, and take the internal consistency between the experts into account when evaluating the performance.

\section{Performance measures}

Given the definitions in section VII-A, we measure the accuracy of our feature detection in terms of the relative sizes of the sets $F P, F N, F C$, and $C C$ with respect to the size of $D$ or $M$. In [40], errors relative to $M$ were considered in all cases. Here, we shall emphasize the following error measures, which are more directly related to the way a matching algorithm treats the detected minutiae:

- $F N / M$ - the proportion of minutiae missed by the algorithm. The complement $1-F N / M$ should be large enough to allow a positive match with the corresponding pair. If we assume that only 6-12 minutiae pairs (i.e. about 10\%) are usually enough to ensure a legally positive identification, we see that relatively high values of $F N / M$ are still admissible.

- $F C / D, F P / D$ - the proportion of errors in classification and detection, within the set of detected minutiae. Certain matching algorithms are very sensitive to even small values of $F P / D$ (noise), and hence minimizing this number becomes a critical task. The importance of $F C / D$ depends on whether the matching algorithm uses type information or not. The complement $C C / D=1-F C / D-F P / D$ measures the proportion of correct minutiae available for matching.

\section{Comparison with traditional enhancement method}

The left table in table I shows the results of feature detection in terms of the abovementioned measures for the proposed enhancement method, referred to as method $G$. For comparison, results are also shown of applying the same feature detection algorithm to the output of a more traditional fingerprint enhancement algorithm (an improved version of [11], referred to as method $F$ ). As can be seen, both methods give a similar, and relatively large, but still acceptable number of false negatives, whereas the remaining performance measures are considerably better for method $G$.

The right table in table I shows corresponding results after introducing an additional post-processing 
TABLE I

Results of feature detection for the proposed method G, ANd a more Classical method F [11].

\begin{tabular}{||c||c|c||c|c||}
\hline \multicolumn{1}{||c||}{ Raw minutiae detection } \\
\hline & \multicolumn{2}{|c||}{ NIST14 } & \multicolumn{2}{c||}{$\mathrm{MM}$} \\
\hline & $\mathrm{F}^{\dagger}$ & $\mathrm{G}^{\dagger}$ & $\mathrm{F}^{\dagger}$ & $\mathrm{G}^{\dagger}$ \\
\hline$F N / M$ & $45.0 \%$ & $49.7 \%$ & $51.8 \%$ & $37.6 \%$ \\
\hline$F P / D$ & $39.0 \%$ & $28.6 \%$ & $33.1 \%$ & $19.4 \%$ \\
$F C / D$ & $16.2 \%$ & $12.9 \%$ & $21.9 \%$ & $9.8 \%$ \\
$C C / D$ & $44.8 \%$ & $58.5 \%$ & $45.1 \%$ & $70.8 \%$ \\
\hline
\end{tabular}

\begin{tabular}{||c||c|c||c|c||}
\hline \multicolumn{4}{||c||}{ After minutiae post-processing } \\
\hline & \multicolumn{2}{|c||}{ NIST14 } & \multicolumn{2}{c||}{$\mathrm{MM}$} \\
\hline & $\mathrm{F}^{\dagger}$ & $\mathrm{G}^{\dagger}$ & $\mathrm{F}^{\dagger}$ & $\mathrm{G}^{\dagger}$ \\
\hline$F N / M$ & $63.8 \%$ & $65.1 \%$ & $67.7 \%$ & $59.3 \%$ \\
\hline$F P / D$ & $10.2 \%$ & $6.2 \%$ & $9.5 \%$ & $3.7 \%$ \\
$F C / D$ & $25.6 \%$ & $17.5 \%$ & $29.2 \%$ & $13.8 \%$ \\
$C C / D$ & $64.3 \%$ & $76.3 \%$ & $61.3 \%$ & $82.5 \%$ \\
\hline
\end{tabular}

step, which drastically reduces the number of false positives at the expense of an increased number of false negatives. We have used a simple set of rules to eliminate "false minutiae", i.e. unlikely configurations in the ridge structure that are most often due to noise [41], [42].

\section{E. Comparison with feature detection methods}

In [40] a similar evaluation was carried out for five other algorithms, referred to as method A [40], B [8], C [45], D [46] and E [14] respectively, using the MM image set: Direct comparison with [40] is, however, not straightforward, for several reasons:

1. Even though the MM image set is the same, the manual features used in [40] (also introduced by an expert) were only available for two images (labeled 1 and 13) out of 14 . Hence, we had to use a different set of manual features (our own), which turned out to differ significantly from the MM feature set.

2. Methods A-E are specialized feature detection and enhancement algorithms, while the evaluation of our enhancement method $\mathrm{G}$ relies on a simple feature detection algorithm to enable comparisons.

3. Methods A-E rely on manual setting of several parameters, notably scale parameters, whereas the proposed method $\mathrm{G}$ is fully automatic.

Table II lists the performance values $\left(A^{*}-E^{*}\right)$ reported in [40] for these five methods applied to the MM image set with its corresponding features. The left table shows corresponding performance values obtained by applying the abovementioned minutiae detection method to the output of our proposed fingerprint enhancement scheme on the MM image set, using our own feature set for evaluation $\left(G^{\dagger}\right)$. The right table shows corresponding values for those two images in the MM image set where the MM feature set is available, and we can thus compare how the evaluation depends upon the feature set used 
TABLE II

Comparison between the performance measures of five methods (A-E) in [40], ANd our method (G). (Note that Performance Values are COMPuted using Different Ground truths.)

\begin{tabular}{|c|c|c|c|c|c|c|c|c|c|c|c|c|c|c|}
\hline & \multicolumn{7}{|c|}{ MM image set } & \multicolumn{7}{|c|}{ MM images 1 and 13} \\
\hline & $\mathrm{A}^{*}$ & $\mathrm{~B}^{*}$ & $\mathrm{C}^{*}$ & $\mathrm{D}^{*}$ & $\mathrm{E}^{*}$ & $\mathrm{G}^{\dagger}$ & $\mathrm{G}^{\dagger *}$ & $\mathrm{~A}^{*}$ & $\mathrm{~B}^{*}$ & $\mathrm{C}^{*}$ & $\mathrm{D}^{*}$ & $\mathrm{E}^{*}$ & $\mathrm{G}^{*}$ & $\mathrm{G}^{\dagger}$ \\
\hline$F N / M$ & $4.5 \%$ & $1.5 \%$ & $5.0 \%$ & $2.3 \%$ & $1.8 \%$ & $37.6 \%$ & $35.3 \%$ & $0.0 \%$ & $0.0 \%$ & $1.9 \%$ & $0.0 \%$ & $0.0 \%$ & $28.0 \%$ & $29.8 \%$ \\
\hline$F P / D$ & $8.2 \%$ & $53.1 \%$ & $68.1 \%$ & $66.7 \%$ & $18.7 \%$ & $19.4 \%$ & $4.1 \%$ & $3.6 \%$ & $57.9 \%$ & $75.4 \%$ & $71.7 \%$ & $18.5 \%$ & $3.6 \%$ & $17.5 \%$ \\
\hline$F C / D$ & $12.5 \%$ & $3.3 \%$ & $2.9 \%$ & $3.2 \%$ & $7.9 \%$ & $9.8 \%$ & $12.5 \%$ & $20.0 \%$ & $3.2 \%$ & $4.7 \%$ & $4.3 \%$ & $13.8 \%$ & $19.6 \%$ & $15.8 \%$ \\
\hline$C C / D$ & $79.3 \%$ & $43.6 \%$ & $28.9 \%$ & $30.1 \%$ & $73.4 \%$ & $70.8 \%$ & $83.5 \%$ & $76.4 \%$ & $38.9 \%$ & $19.9 \%$ & $24.1 \%$ & $67.7 \%$ & $76.8 \%$ & $66.7 \%$ \\
\hline
\end{tabular}

$\left({ }^{*}\right.$ means that the evaluation has been performed with respect to the MM feature set and ${ }^{\dagger}$ with respect to ours). The column labeled $\left(G^{\dagger *}\right)$ gives an extrapolation of the performance values for our feature set to the MM feature set, using the ratio $G^{*} / G^{\dagger}$ for MM images 1 and 13 as normalization.

Although, as mentioned previously, this comparison is not fully fair, we can observe that the performance of the integrated method is competitive compared to the other methods. Our method has a high, but still admissible number of false negatives, but on the other hand the lowest ratio of false positives, and the highest ratio of correctly detected minutiae, which are the most critical parameters.

\section{F. Summary}

Based on these results and the promising results in the evaluations of the ridge width, orientation field and ridgeness measure, we argue that we have with the proposed mechanisms for shape adaptation and automatic scale selection captured essential aspects of fingerprint processing, which should be of interest to developers of systems for automatic fingerprint recognition. Specifically, we believe that there could be a potential for further improvements by combining the proposed scale selection and shape adaptation mechanisms with differential geometric definitions of ridges and minutiae.

\section{ViII. Relations to Previous Work}

Non-linear diffusion. The shape adaptation procedure builds upon work in [15], [24], which was originally aimed at reducing the shape distortions that occur in filter based algorithms for estimating shape from texture and disparity gradients. It also builds upon the non-linear filtering and diffusion ideas proposed in [18], [47] as well as the suggestion in [16] to apply "tensor based diffusion" to fingerprint enhancement. The major difference is that the proposed approach separates the non-linear scale-selection and shape-adaptation procedure from the linear anisotropic diffusion scheme, thus bearing closer relationships to the open-ended suggestion in [21] to regard non-linear scale-space concepts as linear scale-space representations on transformed domains. More recently a non-linear orientation 
diffusion scale-space was proposed [48]. In this context, let us emphasize that the windowed second moment matrix constitutes a well-founded multi-scale representation of orientations [15, section 14.2.3 and 14.4.1] and that it satisfies the diffusion equation $\partial_{s} \mu=\frac{1}{2} \nabla^{2} \mu$.

Algorithmic structure. The general structure of the proposed method resembles the classical structure presented in section I, and used by most classical works on fingerprint image enhancement [3], [8], [9], [10], [11], consisting of an estimation of local image parameters, followed by the application of contextual filters. In this respect, a major difference of the proposed algorithm consists of its iterative coarse to fine structure, where initial coarse estimates lead to a coarsely enhanced image, which is later used to produce more refined estimates and enhanced images, until convergence criteria are achieved.

Multiple directions. The fact that a single orientation cannot be defined at ridge endings and bifurcations has led to several different approaches. In [49] it was proposed to filter along several orientations, by analyzing local Fourier transforms, whereas in [50], [51] it was proposed to apply a set of logical/linear operators for simultaneously applying a directional filter and testing its consistency with the logical preconditions for applying that filter. The proposed method can be considered as a hybrid, since it resembles a filter which continuously varies between a directional filter in regions with clearly defined orientation $(\tilde{Q} \approx 1)$ and an isotropic filter near bifurcations without a single dominant orientation $(\tilde{Q} \approx 0)$. It should also be noted that the methods in [51], [49] are likely to enhance noisy structures like scars, as well as minutiae, since they do not provide an explicit mechanism for distinguishing between the two situations, whereas the proposed method handles this situation by means of a ridgeness measure and automatic scale selection.

Selection of local scales. The proposed method for automatic scale selection bears close relationships to works on wavelet representations [52], and on generalized entropies [53], [54], but most importantly to previous works on feature detection with automatic scale selection [15], [34], [35]. In the specific area of fingerprint analysis, many recent developments stress the need of an automatic scale-selection procedure [40], [50], [14]. In fact, most methods that address scale selection issues in fingerprint analysis are either implicit [49], [55], [12] (and without explicit reports on scale-selection results) or highly discretized [11]. The only exception known to the authors is the recent work in [43], which addresses the problem from a statistical point of view and computes simultaneous estimates of scale and orientation by maximizing a semi-variogram. A main aim of this paper is to contribute to fingerprint analysis by presenting a principled and theoretically well-founded study of the topic of automatic scale selection.

Selection of integration scales. Several works on fingerprint image analysis have previously described alternative methods for tuning the amount of smoothing (integration scale) necessary to obtain reliable orientation estimates. These methods are based on a measure of consistency of the orientation map 
after smoothing, and the smoothing level is selected by maximizing this measure in a coarse-to-fine [14], [7] or fine-to-coarse [17] manner. In this work the integration scale is determined as a function of the local scale and ridgeness. This avoids unreliable estimates near scars and other noisy situations, which can be expected to occur in the methods in [14], [7]. On the other hand, the proposed method has the disadvantage that it gives too large values near cores and deltas. A curvature-dependent scale selection scheme should be able correct for this shortcoming.

Ridgeness measure. The proposed ridgeness measure is closely related to similar measures proposed in [11], [56], but besides being more continuous, the proposed measure was experimentally shown to better discriminate good and poor areas in a fingerprint. This measure also has similar properties as those described in [6], but the details of how that measure is defined were not available. Interestingly, [6] also showed that such a measure can be used with great benefits as a weight in a matching algorithm.

\section{SUmmary AND DISCUSSION}

We have presented a novel methodology for fingerprint image enhancement, and compared it to other approaches, based on a well established set of sample fingerprint images, and using a set of subjective and objective evaluation measures. The experiments led us to the conclusion that the proposed method:

- Gives reasonably accurate and continuous estimates of local ridge width. Whereas this kind of information is of widespread use in fingerprint identification, we know of very little previous work on fingerprint image processing that addresses this problem in a systematic way. Some authors leave this as an open question for future development, while others address it in a slightly ad-hoc manner, tightly intertwined with the enhancement procedure, which results in poor or highly discretized estimates.

- Produces directional fields which are more useful for later stages of an AFIS, since they are continuous both in space and magnitude, the level of detail is adapted to the local image quality, and the reliability is high, since most spurious ridge directions produced by scars or fragmented ridges are corrected, which otherwise mislead methods for fingerprint image enhancement and feature extraction.

- Generates a ridgeness measure with similar properties as other quality measures proposed previously. Such a ridgeness measure was shown to be highly useful for feature extraction, and also for later stages of an AFIS [6]. Furthermore this measure is used to regulate and adapt the diffusion process to the local image quality, an original feature not seen in previous approaches to fingerprint identification.

- Avoids the "checkerboard effect" present in the grey-level output of some spectral based methods.

Thus, this shape adaptation approach addresses key weaknesses of traditional fingerprint image enhancement methods, and should be suitable for use within an AFIS. In particular, the combination of 
the proposed enhancement method with a very simple feature detection module was shown to produce competitive results when compared to other state-of-the-art methods. Hence, it should be possible to integrate the different outputs of the proposed method into a much more reliable feature detection algorithm. Previous work on geometric differential feature detection [35] and gray level ridge counts [43] provide an interesting direction to follow.

More generally, this technique provides an interesting connection between linear and non-linear scalespace approaches, in the sense that the smoothing operation is purely linear and is separated from the non-linear shape adaptation and scale selection procedures. Whereas the more general implications of this approach have not been explored in detail, this technique has the general attractive property of leading to a larger amount of smoothing along edges than across them, while presenting a more conservative behavior around non-edge areas, allowing more complex structures, such as corners or branching points, to be preserved [18], [15]. We believe that non-uniform smoothing schemes based on this general idea should be applicable to a large number of other purposes in image processing.

REFERENCES

[1] A. Almansa and T. Lindeberg, "Enhancement of fingerprint images by shape-adapted scale-space operators," in Gaussian Scale-Space Theory, J. Sporring et al., Eds., Copenhagen, Denmark, May 1996, Kluwer.

[2] A. Almansa and T. Lindeberg, "Fingerprint enhancement by shape adaptation of scale-space operators with automatic scale-selection," Tech. Rep. ISRN KTH/NA/P--98/03--SE, Dept. of Numerical Analysis and Computing Science, KTH, Stockholm, Sweden, Apr. 1998.

[3] K. Asai, Y. Hoshino, N. Yamashita, and S. Hiratsuka, "Fingerprint identification system," in 2nd. USA-Japan Computer Conf., 1975, pp. 30-35.

[4] O. Nakamura, Y. Nagaoka, and T. Minami, "A restoration algorithm of fingerprint images," in Systems and Computers in Japan, 1986, vol. 17:6, p. 31.

[5] R. McCabe, C. Willson, and D. Grubb, Research considerations regarding FBI-IAFIS tasks $E_{3}$ requirements, NISTIR 4892, NIST, July 1992.

[6] F. R. Johannesen, S. Raaschou, O. V. Larsen, and P. Jürgensen, "Using weighted minutiae for fingerprint identification," in Advances in Structural and Syntactical Pattern Recognition. 6th International Workshop, SSPR '96 Proc. 1996, pp. 289-99, Springer-Verlag.

[7] A. Jain, L. Hong, and R. Bolle, "On-line fingerprint verification," IEEE Trans. Pattern Analysis and Machine Intell., vol. 19, no. 4, pp. 302-3013, Apr. 1997.

[8] R.M. Stock and S.W. Swonger, "Development and evaluation of a reader of fingerprint minutiae," Tech. Rep. CAL No. XM-2478-X-1:13-17, Cornell Aeronautical Laboratory, 1969.

[9] K. Sasakawa, F. Isogai, and Ikebata S., "Personal verification system with high tolerance of poor quality fingerprints," in Proc. SPIE, 1990, vol. 1386, pp. 265-272.

[10] B.M. Mehtre, "Fingerprint image analysis for automatic identification," Machine Vision and Applications, vol. 6, pp. 124-139, 1993. 
[11] O. Bergengruen, "Preprocessing of poor quality fingerprint images," in XIV Intl. Conf. of the Chilean Computer Science Society, October 1994.

[12] E. Kaymaz and S. Mitra, "Analysis and matching of degraded and noisy fingerprints," in Applications of Digital Image Processing XV, 1992, vol. 1771 of SPIE, pp. 498-508.

[13] B. G. Sherlock and D. M. Monro, "A model for interpreting fingerprint topology," Pattern Recognition, vol. 26, no. 7, pp. $1047-1055,1993$.

[14] L. O'Gorman and J.V. Nickerson, "An approach to fingerprint filter design," Pattern Recognition, vol. 22, no. 1, pp. $29-38,1989$.

[15] T. Lindeberg, Scale-Space Theory in Computer Vision, Kluwer, 1994.

[16] J. Weickert, "Multiscale texture enhancement," in Proc 6th Int Conf on Computer Analysis of Images and Patterns (Prague Czech). Sep 1995, vol. 970 of Lecture Notes in Computer Science, pp. 230-237, Springer Verlag, Berlin.

[17] N.K. Ratha, K. Karu, S. Chen, and A. Jain, "A real-time matching system for large fingerprint databases," IEEE Trans. Pattern Analysis and Machine Intell., vol. 18, no. 8, pp. 799-813, Aug. 1996.

[18] M. Nitzberg and T. Shiota, "Non-linear image filtering with edge and corner enhancement," in IEEE Trans. on Pattern Analysis and Machine Intell., 1992, vol. 14:8, pp. 826-833.

[19] A. P. Witkin, "Scale-space filtering," in 8th Intl. Conf. on Artificial Intelligence, Karlsruhe, Germany, Aug. 1983, pp. 1019-1022.

[20] J. J. Koenderink, "The structure of images," in Biol. Cyb., 1984, vol. 50, pp. 362-370.

[21] L. M. J. Florack, The Syntactical Structure of Scalar Images, Ph.D. thesis, University of Utrecht, The Netherlands, November 1993.

[22] J. Sporring, M. Nielsen, L. Florack, and P. Johansen, Eds., Gaussian Scale-Space Theory, vol. 8 of Computational Imaging and Vision, Kluwer, 1997.

[23] T. Lindeberg and J. Gårding, "Shape-adapted smoothing in estimation of 3-D depth cues from affine distortions of local 2-D structure," Image and Vision Computing, vol. 15, pp. 415-434, 1997.

[24] T. Lindeberg and J. Gårding, "Shape-adapted smoothing in estimation of 3-D depth cues from affine distortions of local 2-D structure," in Proc. 3rd European Conference on Computer Vision, J.-O. Eklundh, Ed., Stockholm, Sweden, May 1994, vol. 800 of Lecture Notes in Computer Science, pp. 389-400, Springer Verlag, Berlin.

[25] M. A. Föstner and E. Gülch, "A fast operator for detection and precise location of distinct points, corners and centers of circular features," in ISPRS Intercommission Workshop, Interlaken, Switzerland, 1987.

[26] K. Kanatani, "Detection of surface orientation and motion from texture by stereological technique," in J. of Artificial Intell., 1984, vol. 23, pp. 213-237.

[27] L. G. Brown and H. Shvaytser, "Surface orientation from projective foreshortening of isotropic texture autocorrelation," IEEE Trans. Pattern Analysis and Machine Intell., vol. 12, no. 6, pp. 584-588, June 1990.

[28] J. Bigün, G. H. Granlund, and J. Wiklund, "Multidimensional orientation estimation with applications to texture analysis and optical flow," IEEE Trans. Pattern Analysis and Machine Intell., vol. 13, no. 8, pp. 775-790, Aug. 1991.

[29] B. Jähne, Spatio-Temporal Image Processing - Theory and Applications, Springer-Verlag, 1993.

[30] T. Lindeberg and J. Gårding, "Shape from texture from a multi-scale perspective," in Proc. 4th International Conference on Computer Vision, H-H. Nagel, Ed., Berlin, Germany, May 1993, pp. 683-691. Press.

[31] J. Gårding and T. Lindeberg, "Direct computation of shape cues using scale-adapted spatial derivative operators," International Journal of Computer Vision, vol. 17, no. 2, pp. 163-191, 1996. 
[32] T. Lindeberg, "On the axiomatic foundations of linear scale-space," in Gaussian Scale-Space Theory, J. Sporring, et al., Eds., Copenhagen, Denmark, May 1996, Kluwer.

[33] T. Lindeberg, "On scale selection for differential operators," in Proc. 8th Scandinavian Conference on Image Analysis, K. A. Høgdra, B. Braathen, and K. Heia, Eds., Tromsø, Norway, May 1993, pp. 857-866.

[34] T. Lindeberg, "Feature detection with automatic scale selection," International Journal of Computer Vision, vol. 30, no. 2, 1998, in press.

[35] T. Lindeberg, "Edge detection and ridge detection with automatic scale selection," International Journal of Computer Vision, vol. 30, no. 2, 1998, in press.

[36] T. Lindeberg, "On automatic selection of temporal scales in time-casual scale-space," in Proc. AFPAC'97: Algebraic Frames for the Perception-Action Cycle, G. Sommer and J. J. Koenderink, Eds., Kiel, Germany, Sept. 1997, vol. 1315 of Lecture Notes in Computer Science, pp. 94-113, Springer Verlag, Berlin.

[37] T. Lindeberg, "Edge detection and ridge detection with automatic scale selection," in Proc. IEEE Comp. Soc. Conf. on Computer Vision and Pattern Recognition, 1996, San Francisco, California, June 1996, pp. 465-470.

[38] C. I. Watson and C. L. Wilson, "NIST special database 4, fingerprint database," U. S. Department of Commerce, NIST, Advanced Systems Division, Gaithersburg, MD 20899, 1992.

[39] C. I. Watson, "NIST special database 14, mated fingerprint card pairs 2," U. S. Department of Commerce, NIST, Advanced Systems Division, Gaithersburg, MD 20899, Sept. 1993.

[40] D. Maio and D. Maltoni, "Direct gray-scale minutiae detection in fingerprints," IEEE Trans. Pattern Analysis and Machine Intell., vol. 19, no. 1, pp. 27-40, Jan. 1997.

[41] Q. Xiao and H. Raafat, "Fingerprint image postprocessong: A combined statistical and structural approach," Pattern Recognition, vol. 24, no. 10, pp. 985-992, 1991.

[42] G. Sasco and A. Almeida, "Reconocimiento de huellas dactilares," Taller V, Dept. of Numerical Analysis, School of Engineering, University of the Republic of Uruguay, Montevideo, Uruguay, 1995.

[43] K.V. Mardia, A.J. Baczkowski, X. Feng, and T.J. Hainsworth, "Statistical methods for automatic interpretation of digitally scanned finger prints," Pattern Recognition Letters, , no. 18, pp. 1197-1203, 1997.

[44] L. Hong, Y. Wan, and A. Jain, "Fingerprint image enhancement: Algorithm and performance evaluation," IEEE Trans. Pattern Analysis and Machine Intell., vol. 20, no. 8, pp. 777-789, Aug. 1998.

[45] B. Moayer and K.-S. Fu, "A tree system approach for fingerprint pattern recognition," IEEE Trans. Pattern Analysis and Machine Intell., vol. 8, no. 3, pp. 376-387, May 1986.

[46] M.R. Verma, K.K. Majumdar, and B. Chaterjee, "Edge detection in fingerprints," Pattern Recognition, vol. 20, no. 5, pp. 513-523, 1987.

[47] B. M. ter Haar Romeny, Ed., Geometry-Driven Diffusion in Computer Vision, Kluwer, 1994.

[48] P. Perona, "Orientation diffusions," IEEE Trans. Image Processing, vol. 7, no. 3, pp. 457-467, Mar. 1998.

[49] R. Pradenas, "Directional enhancement in the frequency domain of fingerprint images.," in Proc. SPIE, 1997, vol. 2932, pp. 150-160.

[50] L.A. Iverson, Toward discrete geometric models for early vision, Ph.D. thesis, McGill University, Montreal, 1993.

[51] L.A. Iverson and S.W. Zucker, "Logical/linear operators for image curves," IEEE Trans. Pattern Analysis and Machine Intell., vol. 17, no. 10, pp. 982-996, Oct. 1995.

[52] S. Mallat and W.L. Hwang, "Singularity detection and processing with wavelets," IEEE Trans. Pattern Analysis and Machine Intell., vol. 38, no. 2, pp. 617-643, Mar. 1992. 
[53] M. Jägersand, "Saliency maps and attention selection in scale and spatial coordinates: An information theoretic approach," in Proc. 5th Intl. Conf. on Computer Vision, Boston, MA, USA, 1995, pp. 195-202.

[54] J. Sporring and J. Weickert, "On generalized entropies and scale-space," Tech. Rep. DIKU-96/37, ISSN 0107-8283, Univ. of Copenhaguen, Universitetsparken 1, DK-2100 Copenhaguen, Denmark - http://www.diku.dk, Mar. 1997.

[55] B.G. Sherlock, D.M. Monro, and K. Millard, "Algorithm for enhancing fingerprint images," Electronic Letters, vol. 28, no. 18, pp. 1720-1, Aug. 1992.

[56] G. T. Candela, P. J. Grother, C. I. Watson, R. A. Wilkinson, and C. L. Wilson, "PCASYS - A pattern-level classification automation system for fingerprints," U.S. Department of Commerce, NIST, Visual Image Processing Group, Gaithersburg, MD 20899, 1995.
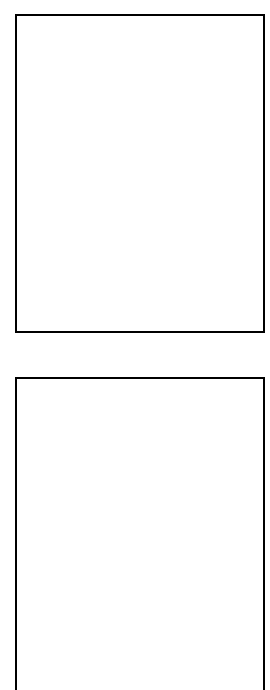

Andrés Almansa received his Engineer, and M.Sc. degrees in Computer Science both from the University of the Republic of Uruguay (UR), in 1995 and 1998 respectively. He is currently an Adjunct Professor at the School of Engineering at UR, where he teaches computer science and numerical analysis. Since 1993, he works on research and consultancy agreements on fingerprint image processing.

and discrete scale-space theory, as well as to the application of these theories to computer vision problems. Specifically, he has developed principles for automatic scale selection, methodologies for extracting salient image structures, and theories for multi-scale shape estimation. He is author of the book "Scale-Space Theory in Computer Vision". 